\title{
CAPÍTULOX
}

\section{Información y educación para la sostenibilidad de espacios ambientales protegidos en Colombia}

\author{
Johann Pirela Morillo* \\ Yamely Almarza Franco****
}

* Licenciado en Bibliotecología y Archivología. (Universidad del Zulia-Venezuela), Magíster Scientiarum en Museología. (Universidad Nacional Experimental "Francisco de Miranda", 2000), Magíster Scientiarum en Educación. Mención: Currículo, 2016, Doctor en Ciencias Humanas (Universidad del Zulia), Postdoctorado en Ciencias Humanas (Universidad del Zulia-Venezuela). Líder del grupo de investigación: Información, desarrollo y sociedad. Docente e investigador de pregrado y postgrado. Actualmente docente investigador del Programa de Sistemas de Información, Bibliotecología y Archivística, Universidad de La Salle-Colombia

** Licenciada en Bibliotecología y Archivología (Universidad del Zulia-Venezuela), Magíster Scientiarum en Ciencias de la Comunicación. Mención: Nuevas Tecnologías (Universidad del Zulia-Venezuela), Doctora en Patrimonio Cultural (Universidad Latinoamericana y del Caribe ULAC). Docente e investigadora, adscrita al Programa de Sistemas de Información, Bibliotecología y Archivística, Universidad de La Salle, Colombia. 


\section{Introducción}

El documento sobre los resultados de la Cumbre del Milenio de Naciones Unidas subraya, diversos principios y estrategias, que deben considerarse por parte de las instituciones educativas, culturales y sociales para avanzar en la construcción de un desarrollo sostenible, el cual reconoce el potencial del acceso a la información: sus medios, fuentes y tecnologías y el fortalecimiento de los sistemas educativos para el logro de los objetivos y metas trazadas. En este sentido, los 17 Objetivos de Desarrollo Sostenible con sus 169 metas demuestran la naturaleza, alcance y preponderancia de esta agenda, en la cual se comprometen no solo las capacidades de diversos estamentos de la sociedad organizada, sino que se asume también como un llamado a la instalación de una cultura de protección, defensa, saneamiento y protección del medioambiente, sobre todo los espacios ambientales protegidos que son fuentes esenciales para el conocimiento, la investigación y la apropiación con miras a construir una conciencia planetaria con visión de integralidad y sostenibilidad.

Siguiendo esta línea, la sostenibilidad del medioambiente se asume como un asunto urgente que debe abordarse desde estrategias informativas y educativas que permitan a los ciudadanos construir los referentes de sentido que favorecerán la configuración progresiva de una consciencia crítica, ecológica y comprometida con la salvaguardia y defensa de espacios ambientales protegidos, en virtud de sus valores científicos, sociales y culturales, reconociendo en tales espacios territorios complejos en los cuales interactúan las personas con sus itinerarios histórico-sociales y con su estructuración cultural.

En el marco de estos argumentos, este capítulo presenta las visiones informacional y educativa, mediante las cuales se deben abordar los espacios ambientales protegidos en Colombia, y con ello, avanzar hacia procesos de sostenibilidad medioambiental que reconozcan el enorme potencial que tienen tales espacios para contribuir con el aprendizaje sobre variables e indicadores esenciales de los territorios, desde cuyos ámbitos se deriven narrativas y se fijen las posiciones reflexivas y críticas que conduzcan hacia la construcción y fortalecimiento de los procesos de identidad, con lo cual se avanzará hacia elevados niveles de calidad de vida mucho más sostenibles y 
en sintonía con los requerimientos que plantea el desarrollo humano integral.

\section{Información y sostenibilidad}

La información es el recurso más valioso de los países, y por ende, no escapa al contexto de la sostenibilidad, esto se deriva de la evolución de la sociedad post-industrial a la sociedad de la información que abre oportunidades para el establecimiento del desarrollo sostenible que involucra mayor compromiso de las comunidades. Se presenta un énfasis en la oferta de servicios, aumentan las inversiones y la población es más activa y participativa, haciendo que los recursos humanos sean un factor de desarrollo haciendo uso de la disponibilidad de los recursos naturales locales. En este contexto, el medio ambiente se valoriza mucho más y se relaciona de manera estrecha con los procesos de desarrollo en la necesidad de alcanzar una conservación dinámica del medio ambiente y de los recursos naturales.

En este nuevo panorama mundial, la información se convierte en un recurso transversal que circula en diversas direcciones ya que se puede recibir, emitir y transmitir de manera simultánea a grandes escalas a través del internet, lo cual ha abierto también nuevos espacios de interacción como son las redes sociales, que posibilitan la interacción de las personas, comunidades y empresas que comparten intereses. Dicha transversalidad de la cual está impregnada la información la hace adquirir un poder sin precedentes ya que se encuentra dispersa, distribuida y descentralizada que a su vez permite una mayor participación ciudadana e inclusión de la población desprotegida.

Así, las poblaciones locales tienen la posibilidad de generar recursos para su bienestar, convirtiéndose en actores de su propio desarrollo y de la conservación de sus recursos y bienes culturales para crear un mejor futuro. De tal manera, que el dominio cultural deviene de las acciones de capacitación permanente para el aprovechamiento de sus potenciales y lograr una mayor autonomía en las decisiones que tomen. La información como recurso de los países, debe ser asimilada por las comunidades en función de la erradicación de la pobreza y lograr un desarrollo que dignifique las poblaciones en torno a 
su cultura. En este particular, los profesionales de la información y las organizaciones de conocimiento cumplen una labor fundamental en la difusión y apropiación crítica de la información sobre los espacios protegidos pues de ello depende que se valoricen los recursos naturales, se conserven y utilicen en función del desarrollo sostenible. La oferta de servicios y productos centrados en los recursos naturales, áreas protegidas y la cultura en general, logra una mayor integración de los pueblos con sus riquezas. Esos contenidos establecidos como mecanismos para el disfrute, interpretación y valorización, no sólo involucra la inclusión sino que también permite que se den procesos de apropiación crítica de los contenidos en torno de la cultura y el patrimonio como garantía de la conservación de los espacios protegidos y de la cultura.

Hoy, es posible que las comunidades se acerquen a través de las Tecnologías de la Información y Comunicación (TICS), ya no existen barreras limítrofes, temporales o espaciales para que se integren las culturas similares en proyectos de gestión cultural que permitan el desarrollo común, integrando a las poblaciones desprotegidas, haciendo posible la inclusión y participación de todos en pro del desarrollo sostenible de las naciones. No es una limitante el acceso a la información y este hecho acrecienta la simbiosis entre lo urbano y rural, ya con mayores oportunidades y sin ser necesario el desplazamiento desde al campo a la ciudad. Ahora el contexto rural adquiere valor pues la capacitación puede darse a través de la Internet y los habitantes rurales son más receptivos a tales iniciativas. Los espacios naturales protegidos ofrecen la oportunidad para la generación de modelos de desarrollo sostenible, como zonas de influencia socioeconómica con la tendencia de romper el aislamiento territorial para generar y resaltar una mayor preocupación por el bienestar de la población que vive en ellos.

De tal manera, que el turismo en esta nueva realidad adquiere gran valor en relación con el desarrollo sostenible, haciendo más fuerte el sector cultural y económico que dan paso a nuevos puestos laborales que integran las familias como empresas, pero a la vez, es un sector que involucra una gestión más profunda porque implica en paralelo la conservación de los espacios protegidos, lo cual diferencia este tipo de turismo social de masas que tiene como característica la calidad y protección de la cultura y medio ambiente. 
El paradigma estático de la conservación ha quedado ya obsoleto en gran medida, y actualmente se reconoce universalmente que no es funcional incluso para la conservación de las especies singulares para las que precisamente se crearon y acotaron muchos de los espacios naturales protegidos. (Foronda Robles, 2012, p.25)

Avanzar en torno a esta posibilidad requiere el compromiso de los gobiernos, las administraciones y la integración y participación ciudadanía, a su vez, estará determinado por las características socioeconómicas de los municipios y estados en los que se sitúan estos espacios protegidos que regularmente son zonas con carencias económicas, pero que pueden convertirse en un motor para generar nuevas formas de rentabilidad, de desarrollo social y de conservación del patrimonio natural y cultural.

Estas relaciones que se establecen en torno a la información permiten el mayor aprovechamiento de los recursos naturales en pro del turismo ecológico y cultural que puede ser mayormente explotado a través de las TICS. Esta nueva manera de hacer turismo es una manera de estimular el desarrollo económico en sintonía con la conservación y el enriquecimiento de la cultura, la naturaleza y sus diversidades. El objetivo se orienta al aumento del bienestar de los pueblos, la inclusión social y la participación, lo cual a su vez, aumenta la apropiación, valorización y protección del patrimonio cultural y natural, al mismo tiempo que posibilita que las comunidades se encuentren y desarrollen en torno a sus riquezas, legados, cultura y entorno, hecho posible en una sociedad basada en la información y la interactividad que permiten las TICS para los intercambios posible en torno al conocimiento colectivo. Así, los planes orientados hacia el desarrollo sostenible persiguen el mejoramiento de la calidad de vida de las poblaciones desprotegidas por encontrarse en territorios rurales aislados donde con frecuencia se encuentran los espacios protegidos pero que se convierten en un importante recurso para el desarrollo económico y local.

\section{Educación y sostenibilidad}

Además de la información como proceso y como despliegue de operaciones que permiten sistematizar y articular datos, disponibles desde sistemas que integren contenidos sobre los espacios ambientales protegidos en Colombia; se incluye otro componente, 
que consideramos de vital importancia para desarrollar acciones que estén orientadas hacia el logro de competencias y de actitudes que permitan la configuración de una cultura para la preservación y conservación del medio-ambiente, no solo en su dimensión de espacio físico y ambiental, sino también considerando su potencialidad como territorio de interacciones entre lo ecológico, cultural y social. De esta forma, se plantea la educación como un conjunto de acciones articuladas, enfocadas a formar la consciencia crítica necesaria para un uso sustentable de los recursos ambientales, que aporte también el arsenal cognitivo para generar otros tipos de dinámicas que tienen que ver con la construcción de los saberes necesarios en torno a lo ambiental, en una perspectiva de protección y defensa del patrimonio natural, como parte indisoluble de los procesos de identidad nacional.

En este sentido, y luego de realizar una investigación acerca de la naturaleza y el alcance que se le da al concepto de educación para la sostenibilidad, puede apreciarse que tal expresión hunde sus raíces en las definiciones iniciales de educación ambiental, noción que reconocía el potencial del medio-ambiente y su protección como variable fundamental del desarrollo integral y humano, que debía incorporarse como una dimensión fundamental de los procesos de formación integral. Desde esta perspectiva, es tal vez, que organismos internacionales como UNESCO (2006) y más reciente (2014), hayan estado proponiendo una serie de principios y estrategias sustentadas en criterios que aluden a la integración necesaria entre "la educación ambiental", "la educación global", "la educación económica", "la educación para el desarrollo", "la educación multicultural", "la educación para la conservación", "la educación sobre el cambio global” y otras. Expresiones éstas que convocan la necesaria articulación de procesos educativos como base de la construcción de un presente y un futuro sostenible.

El concepto de educación para la sostenibilidad implica, un conjunto amplio de variables e indicadores que integran diversas aristas, vinculadas con las expresiones señaladas, razón por la cual es un concepto que apela a una serie de prácticas presentes en las agendas de las tendencias educativas, las cuales convocan que todos los niveles y modalidades de los diferentes sistemas educativos a escala global, tracen los itinerarios requeridos para que estos temas sean atendidos desde los procesos formativos, tanto en ámbitos formales como en los no formales. El foco de la propuesta, debe estar en 
el diseño y puesta en marcha de planes y estrategias apoyadas en la transversalidad curricular, debido a que no se trata solamente de incluir asignaturas y espacios académicos en los cuales se aborden las problemáticas relacionadas con el conocimiento, disfrute responsable y conservación de los espacios ambientales protegidos, sino que se requiere que dichos temas hagan parte de las estrategias formativas en diferentes asignaturas. El tema, más que una asignatura adicional que se incorpora en el currículo, debe ser abordado como eje transversal, para fomentar una conciencia ética y crítica en torno a la valorización y protección del medioambiente.

En el marco de estos argumentos expresados, se considera conveniente también mencionar que durante la 63ª Asamblea General de Naciones Unidas, celebrada en 2003, la Comisión Alemana para la UNESCO aprobó la Declaración de Hamburgo, en la cual se hace un llamado a todos los grupos sociales organizados, instituciones educativas, culturales y demás estamentos de la sociedad civil, a conformar un bloque común en pro de establecer una alianza para el aprendizaje de la sostenibilidad. El objetivo de la Declaración era la elaboración conjunta de un plan de actuación para la década, así como la formulación de programas y mecanismos de coordinación para su puesta en práctica. Características como: la interdisciplinariedad y globalidad, la centralidad en los valores, el pensamiento crítico para la solución de problemas, el abordaje multi-metodológico, participación en la toma de decisiones e importancia de lo local constituyen las características más relevantes de la educación para la sostenibilidad, de acuerdo a lo señalado por la UNESCO (2006), frente a las cuales se precisa destacar que la educación para un desarrollo sostenible se enmarca en una perspectiva de formación continua, lo cual a su vez correlaciona con el concepto de educación a lo largo de la vida, aquella que rebasa los espacios de educación formal e institucional.

La educación para la sostenibilidad debe propiciar el desarrollo de estructuras cognitivas y afectivas favorables para que se instale la cultura de la sostenibilidad en diversos estamentos sociales, mediante estrategias que tienen que ver con el aprendizaje de lo sensible y de los elementos que estructuran el ser social y cultural, así como la pertenencia al territorio. La educación para la sostenibilidad se apoya en lo metodológico en opciones didácticas creativas e interactivas, que busquen en todo momento la movilización de saberes, de pensamientos divergentes y de esquemas cognitivos que valoren lo local, 
posibilitando también la generación de una cultura para la preservación, conservación y protección del ambiente. Según Gutiérrez-Pérez y Pozo (2005), la educación para la sostenibilidad debe movilizar la conciencia colectiva y generar pautas para transitar hacia procesos de cambio de las estructuras con las cuales se realizan la apropiación de la información y del conocimiento sobre los espacios ambientales protegidos. Además de cuestiones de tipo cognitivo, debe partir de la concientización y participación como acciones que deben articularse también con el compromiso por la protección y defensa del medio-ambiente, en tanto que patrimonio natural. Estos planteamientos ya habían sido referidos por González Gaudiano (1997), quien enfatiza en la necesaria inclusión y orientación hacia los diferentes grupos y sectores sociales deben constituirse como un componente articulador y favorecedor, tanto en la generación de tecnologías alternativas, de modos de aprovechamiento racional de los recursos y de nuevas propuestas legislativas, como de modificaciones en las formas tradicionales de planificación.

Por otro lado, Ruiz, Barraza y Ceja (2009), desde el contexto mexicano, realizaron un estudio sobre los contenidos presentes en el currículo de bachillerato, encontrando resultados como: 1) la falta de participación de los jóvenes en las clases, a pesar de la motivación de los maestros al impartirlas, y 2) el abordaje limitado de los contenidos ambientales y locales en las materias que no son propias de las "ciencias naturales". Además, los resultados indican que las prácticas docentes se limitaron a los enfoques tradicionales y técnicos, en los que la prioridad y el énfasis es la transmisión de conocimientos de una determinada disciplina, sin considerar contenidos relevantes a nivel ambiental ni social de cada comunidad. Ante lo cual, se requiere que la educación para la sostenibilidad se asuma desde un proceso de formación continua, que lleve a construir de forma colectiva una ciudadanía informada e implicada, que disponga de herramientas creativas para la resolución de los problemas, una cultura científica y social, y el compromiso de protagonizar actuaciones responsables, tanto individuales como colectivas.

Tomando en cuenta estos principios y estrategias, propias de la educación para la sostenibilidad, Leal (2009), señaló que la forma para garantizar un futuro viable desde el punto de vista ecológico y próspero en lo económico, se deben articular estrategias educativas dirigidas al reconocimiento del potencial que tiene la educación para 
apalancar procesos de formación de consciencias críticas, preparadas para asumir el medio-ambiente en sus diversos componentes e indicadores, con lo cual se activarán las sinergias necesarias entre el aula y la empresa, y entre las escuelas y las comunidades. Más recientemente, la UNESCO (2014), ha estado exhortando hacia la generación de múltiples formas y alternativas que permitan lograr los objetivos de desarrollo sostenible propuestos para 2015 y el período posterior. El Ilamado de la UNESCO enfatiza en la concepción de la educación no solo en su dimensión de derecho humano fundamental, sino en su alta potencialidad para impulsar el desarrollo sostenible. La educación permite que las personas, en particular las mujeres, vivan y aspiren a tener una existencia sana, plena de sentido, creativa y resiliente, con lo cual se refuerza la idea su voz en los asuntos comunitarios, nacionales e internacionales. Les brinda nuevas oportunidades laborales y vías de movilidad social. En pocas palabras, la educación tiene efectos importantes en muchos otros sectores y merece ser un pilar esencial del marco para el desarrollo después de 2015.

Tratando de elaborar una síntesis sobre los planteamientos analizados y tomando en cuenta el Decreto 2372 de 2010, definido desde el contexto colombiano, es importante destacar el uso sostenible de las áreas protegidas como la utilización de los componentes de la biodiversidad de un modo y a un ritmo que no ocasione su disminución o degradación a largo plazo alterando los atributos básicos de composición, estructura y función, con lo cual se mantienen las posibilidades de éstas para satisfacer las necesidades y las aspiraciones de generaciones actuales y futuras. La preservación de estos espacios se define como el mantenimiento de la composición, estructura y función de la biodiversidad, conforme su dinámica natural y evitando al máximo la intervención humana y sus efectos. Dentro de los usos permitidos de los espacios ambientales protegidos las actividades que se tienen: la investigación, monitoreo o educación ambiental que aumentan la información, el conocimiento, el intercambio de saberes, la sensibilidad y conciencia frente a temas ambientales y la comprensión de los valores y funciones naturales, sociales y culturales de la biodiversidad. (Artículo 35, inciso c, Decreto 2372 de 2010).

\section{Espacios ambientales protegidos en Colombia.}

Éstos, son ricos en biodiversidad, lo cual es un atractivo turístico para quienes desean estar en contacto directo con la naturaleza. Con 
más de 1876 especies de aves, Colombia tiene mucho que ofrecer. En sus costas, pueden verse las ballenas jorobadas que entre julio y noviembre dan a luz a sus crías. En cuanto a los parques nacionales, éstos se convierten en un eje relevante para la gestión turística, siempre considerando que estas actividades generen el menor impacto posible en los ecosistemas. De acuerdo con diversos estudios realizados sobre la riqueza de la biodiversidad se reconoce que Colombia es uno de los países más diversos del mundo desde el aspecto de su biología y cultura. En tal sentido, se señala que el país "cuenta con unos 54 espacios protegidos y una gran variedad de espacios protegidos de índole provincial, municipal y privados". (P. Feinsinger, C. Pozzi, C. Trucco, R.L. Cuellar, A. Laina, M. Cañizares, A. Noss 7, 2010, p.105)

De igual manera, se ha considerado la capacitación de los funcionarios que laboran en los parques nacionales, así como de los indígenas que habitan en las zonas protegidas. Hoy, los funcionarios capacitados indagan en sus espacios protegidos promoviendo a la vez la incorporación de la comunidad a estas actividades. El papel fundamental de la comunidad colombiana en este particular es asegurar que las áreas protegidas no sean espacios inaccesibles, conservándolas al mismo tiempo, lo cual ha dado paso, a una política nacional apoyada en altos niveles de conservación.

Con el paso del tiempo ha sido necesario establecer relaciones entre desarrollo económico y medio ambiente, el cual se pone de manifiesto principalmente a partir de los años 80 cuando triunfa la idea que, los Espacios Naturales Protegidos (ENP) no son simples reductos de naturaleza, a partir del informe Brundtland (1987) o la Cumbre de Río (1992). (Foronda Robles, 2012, p. 25)

Este hecho ha fomentado la participación de la comunidad en las actividades sociales, desarrollando sentido de pertenencia e inclusión, aspectos relevantes para el bienestar social; lo contrario, sería un verdadero problema para el desarrollo sostenible basado en los espacios protegidos, pues se constituyen en elementos que revitalizan la economía de las zonas rurales. Lo que se hace necesario es que la participación de la población sea en el marco de la organización y planificación de tareas que se vean apoyadas por un equipo de trabajo interdisciplinario. 
Debe resaltarse el sentido ecoturístico que denota el bajo impacto ambiental en las zonas protegidas donde se desarrollen actividades turísticas, a su vez, abre nuevos escenarios para la participación y la reactivación de las economías locales. El turismo ecológico se valora como una estrategia de preservación de la identidad y aprovechamiento de los recursos locales y desarrollo de los pueblos indígenas y poblaciones desprotegidas. Todo apunta hacia crear efectivas interrelaciones entre las actividades turísticas y los espacios protegidos, esto se hace posible a través de la planificación y gestión de los recursos naturales para que se conviertan en fuentes generadoras de ingresos basados en un marco legislativo nutrido que además involucre la educación ecológica. Al respecto, la Declaración de la Haya adoptada por la Conferencia Interparlamentaria sobre Turismo el 14 de abril de 1989 de la Organización Mundial del Turismo, destaca "la integridad del medio natural, cultural y humano es condición fundamental para el desarrollo del turismo, una gestión racional puede contribuir, a la protección y mejora del entorno físico del patrimonio cultural, así como el aumento de la calidad de vida". (Unión interparlamentaria, 1989, p. 5).

De tal manera que se pueden considerar en Colombia como espacios ambientales protegidos que se pueden aprovechar con fines turísticos, educativos y culturales, las zonas identificadas en el Cuadro No. 1. En este sentido, son muchas y diversas las posibilidades para el turismo ecológico y de investigación que existen en la cantidad de parques nacionales y zonas protegidas del territorio colombiano, entre las que destacan:

Cuadro 1. Espacios ambientales protegidos en Colombia

\begin{tabular}{|c|c|c|c|c|}
\hline Denominación & Año & Región & Departamento & Extensión \\
\hline $\begin{array}{l}\text { Vía Parque Isla de } \\
\text { Salamanca }\end{array}$ & 1964 & Costa del Caribe & Magdalena & 56200 \\
\hline $\begin{array}{l}\text { Santuario de fauna y flora } \\
\text { Plantas Medicinales Orito } \\
\text { Ingi-Andé }\end{array}$ & 2008 & Amazonía & $\begin{array}{l}\text { Nariño } \\
\text { Putumayo }\end{array}$ & 10204 \\
\hline $\begin{array}{l}\text { Santuario de fauna y flora } \\
\text { Otún Quimbaya }\end{array}$ & 1996 & $\begin{array}{c}\text { Andes (Cordillera } \\
\text { Central) }\end{array}$ & Risaralda & 489 \\
\hline $\begin{array}{l}\text { Santuario de fauna y flora } \\
\text { Malpelo }\end{array}$ & 1995 & Insular & Valle del Cauca & $120^{5}$ \\
\hline $\begin{array}{l}\text { Santuario de fauna y flora } \\
\text { los Flamencos }\end{array}$ & 1977 & Costa del Caribe & La Guajira & 7682 \\
\hline
\end{tabular}




\begin{tabular}{|c|c|c|c|c|}
\hline Denominación & Año & Región & Departamento & Extensión \\
\hline $\begin{array}{l}\text { Santuario de fauna y flora } \\
\text { Los Colorados }\end{array}$ & 1977 & Costa del Caribe & Bolívar & 1000 \\
\hline $\begin{array}{l}\text { Santuario de fauna y flora } \\
\text { Isla de La Corota }\end{array}$ & 1977 & $\begin{array}{c}\text { Andes (Cordillera } \\
\text { Central) }\end{array}$ & Nariño & 16 \\
\hline $\begin{array}{l}\text { Santuario de fauna y flora } \\
\text { Iguaque }\end{array}$ & 1977 & $\begin{array}{c}\text { Andes (Cordillera } \\
\text { Oriental) }\end{array}$ & Boyacá & 6750 \\
\hline $\begin{array}{l}\text { Santuario de fauna y flora } \\
\text { Guanentá Alto Río Fonce }\end{array}$ & 1993 & $\begin{array}{c}\text { Andes (Cordillera } \\
\text { Oriental) }\end{array}$ & $\begin{array}{l}\text { Boyacá } \\
\text { Santander }\end{array}$ & 10429 \\
\hline $\begin{array}{l}\text { Santuario de fauna y flora } \\
\text { Galeras }\end{array}$ & 1985 & $\begin{array}{l}\text { Andes (Nudo de } \\
\text { los Pastos) }\end{array}$ & Nariño & 7615 \\
\hline $\begin{array}{l}\text { Santuario de fauna y flora El } \\
\text { Corchal El mono Hernández }\end{array}$ & 2002 & Costa del Caribe & $\begin{array}{l}\text { Sucre } \\
\text { Bolívar }\end{array}$ & 3850 \\
\hline $\begin{array}{l}\text { Santuario de fauna y flora } \\
\text { Ciénaga Grande de Santa } \\
\text { Marta }\end{array}$ & 1977 & Costa del Caribe & Magdalena & 26810 \\
\hline $\begin{array}{l}\text { Santuario de fauna y flora } \\
\text { Acandí, Playón y Playona }\end{array}$ & 2013 & Costa del Caribe & Chocó & 26232 \\
\hline $\begin{array}{l}\text { Parque nacional natural } \\
\text { Cueva de los Guácharos }\end{array}$ & 1960 & $\begin{array}{c}\text { Andes (Cordillera } \\
\text { Oriental) }\end{array}$ & Huila & 9000 \\
\hline $\begin{array}{l}\text { Parque nacional natural } \\
\text { Tayrona }\end{array}$ & 1964 & Costa del Caribe & Magdalena & 15000 \\
\hline $\begin{array}{l}\text { Parque nacional natural } \\
\text { Puracé }\end{array}$ & 1968 & $\begin{array}{c}\text { Andes (Cordillera } \\
\text { Central) }\end{array}$ & $\begin{array}{c}\text { Cauca } \\
\text { Huila }\end{array}$ & 83000 \\
\hline $\begin{array}{l}\text { Parque nacional natural } \\
\text { Farallones de Cali }\end{array}$ & 1968 & $\begin{array}{c}\text { Andes (Cordillera } \\
\text { Occidental) }\end{array}$ & Valle del Cauca & 150000 \\
\hline $\begin{array}{l}\text { Parque nacional natural El } \\
\text { Tuparro }\end{array}$ & 1970 & Orinoquía & Vichada & 548000 \\
\hline $\begin{array}{l}\text { Parque nacional natural } \\
\text { Sierra de La Macarena }\end{array}$ & 1971 & Orinoquía & $\begin{array}{l}\text { Meta } \\
\text { Guaviare }\end{array}$ & 620000 \\
\hline $\begin{array}{l}\text { Parque nacional natural Los } \\
\text { Nevados }\end{array}$ & 1973 & $\begin{array}{l}\text { Andes (Cordillera } \\
\text { Central) }\end{array}$ & $\begin{array}{l}\text { Risaralda } \\
\text { Tolima } \\
\text { Caldas } \\
\text { Quindío }\end{array}$ & 38000 \\
\hline $\begin{array}{l}\text { Parque nacional natural Las } \\
\text { Orquídeas }\end{array}$ & 1973 & $\begin{array}{l}\text { Andes (Cordillera } \\
\text { Occidental) }\end{array}$ & Antioquia & 32000 \\
\hline $\begin{array}{l}\text { Parque nacional natural de } \\
\text { Los Katíos }\end{array}$ & 1973 & Costa del Pacífico & $\begin{array}{l}\text { Chocó } \\
\text { Antioquia }\end{array}$ & 72000 \\
\hline $\begin{array}{l}\text { Parque nacional natural } \\
\text { Amacayacu }\end{array}$ & 1975 & Amazonía & Amazonas & 293500 \\
\hline $\begin{array}{l}\text { Parque nacional natural } \\
\text { Tamá }\end{array}$ & 1977 & $\begin{array}{c}\text { Andes (Cordillera } \\
\text { Oriental) }\end{array}$ & $\begin{array}{l}\text { Norte de } \\
\text { Santander }\end{array}$ & 15000 \\
\hline $\begin{array}{l}\text { Parque nacional natural } \\
\text { Sumapaz }\end{array}$ & 1977 & $\begin{array}{l}\text { Andes (Cordillera } \\
\text { Oriental) }\end{array}$ & $\begin{array}{c}\text { Meta } \\
\text { Cundinamarca } \\
\text { Huila }\end{array}$ & 154000 \\
\hline
\end{tabular}




\begin{tabular}{|c|c|c|c|c|}
\hline Denominación & Año & Región & Departamento & Extensión \\
\hline $\begin{array}{l}\text { Parque nacional natural } \\
\text { Sanquianga }\end{array}$ & 1977 & Costa del Pacífico & Nariño & 80000 \\
\hline $\begin{array}{l}\text { Parque nacional natural } \\
\text { Pisba }\end{array}$ & 1977 & $\begin{array}{c}\text { Andes (Cordillera } \\
\text { Oriental) }\end{array}$ & Boyacá & 45000 \\
\hline $\begin{array}{l}\text { Parque nacional natural } \\
\text { Paramillo }\end{array}$ & 1977 & $\begin{array}{l}\text { Andes (Cordillera } \\
\text { Occidental) }\end{array}$ & $\begin{array}{l}\text { Córdoba } \\
\text { Antioquia }\end{array}$ & 460000 \\
\hline $\begin{array}{l}\text { Parque nacional natural } \\
\text { Nevado del Huila }\end{array}$ & 1977 & $\begin{array}{l}\text { Andes (Cordillera } \\
\text { Central) }\end{array}$ & $\begin{array}{l}\text { Huila } \\
\text { Tolima } \\
\text { Cauca }\end{array}$ & 158000 \\
\hline $\begin{array}{l}\text { Parque nacional natural } \\
\text { Munchique }\end{array}$ & 1977 & $\begin{array}{c}\text { Andes (Cordillera } \\
\text { Occidental) }\end{array}$ & Cauca & 44000 \\
\hline $\begin{array}{l}\text { Parque nacional natural Las } \\
\text { Hermosas }\end{array}$ & 1977 & $\begin{array}{l}\text { Andes (Cordillera } \\
\text { Central) }\end{array}$ & $\begin{array}{l}\text { Tolima } \\
\text { Valle del } \\
\text { Cauca }\end{array}$ & 125000 \\
\hline $\begin{array}{l}\text { Parque nacional natural Isla } \\
\text { Gorgona }\end{array}$ & 1983 & Insular & Cauca & \\
\hline $\begin{array}{l}\text { Parque nacional natural La } \\
\text { Paya }\end{array}$ & 1984 & Amazonía & Putumayo & \\
\hline $\begin{array}{l}\text { Parque nacional natural } \\
\text { Cahuinarí }\end{array}$ & 1986 & Amazonía & Amazonas & \\
\hline $\begin{array}{l}\text { Parque nacional natural } \\
\text { Ensenada de Utría }\end{array}$ & 1987 & Costa del Pacífico & Chocó & \\
\hline $\begin{array}{l}\text { Parque nacional natural } \\
\text { Tatamá }\end{array}$ & 1987 & $\begin{array}{l}\text { Andes (Cordillera } \\
\text { Occidental) }\end{array}$ & $\begin{array}{l}\text { Chocó } \\
\text { Risaralda } \\
\text { Valle del } \\
\text { Cauca }\end{array}$ & \\
\hline $\begin{array}{l}\text { Reserva nacional natural } \\
\text { Puinawai }\end{array}$ & 1989 & Amazonía & Guainía & \\
\hline $\begin{array}{l}\text { Reserva nacional natural } \\
\text { Nukak }\end{array}$ & 1989 & Amazonía & $\begin{array}{l}\text { Guaviare } \\
\text { Vaupés }\end{array}$ & \\
\hline $\begin{array}{l}\text { Parque nacional natural } \\
\text { Tinigua }\end{array}$ & 1989 & Amazonía & Meta & \\
\hline $\begin{array}{l}\text { Parque nacional natural } \\
\text { Catatumbo Barí }\end{array}$ & 1989 & $\begin{array}{c}\text { Andes (Cordillera } \\
\text { Oriental) }\end{array}$ & $\begin{array}{l}\text { Norte de } \\
\text { Santander }\end{array}$ & \\
\hline $\begin{array}{l}\text { Parque nacional natural } \\
\text { Sierra de Chiribiquete }\end{array}$ & 1989 & Amazonía & $\begin{array}{l}\text { Caquetá } \\
\text { Guaviare }\end{array}$ & \\
\hline $\begin{array}{l}\text { Parque nacional natural Old } \\
\text { Providence McBean Lagoon }\end{array}$ & 1995 & Insular & $\begin{array}{l}\text { San Andrés y } \\
\text { Providencia }\end{array}$ & \\
\hline $\begin{array}{l}\text { Parque nacional natural Río } \\
\text { Puré }\end{array}$ & 2002 & Amazonía & Amazonas & \\
\hline $\begin{array}{l}\text { Parque nacional natural Alto } \\
\text { Fragua Indi-Wasi }\end{array}$ & 2002 & Amazonía & Caquetá & \\
\hline $\begin{array}{l}\text { Parque nacional natural } \\
\text { Serranía de los Yariguíes }\end{array}$ & 2005 & $\begin{array}{c}\text { Andes (Cordillera } \\
\text { Oriental) }\end{array}$ & Santander & \\
\hline
\end{tabular}




\begin{tabular}{|c|c|c|c|c|}
\hline Denominación & Año & Región & Departamento & Extensión \\
\hline $\begin{array}{l}\text { Parque nacional natural } \\
\text { Selva de Florencia }\end{array}$ & 2005 & $\begin{array}{c}\text { Andes (Cordillera } \\
\text { Central) }\end{array}$ & Caldas & \\
\hline $\begin{array}{l}\text { Parque nacional } \\
\text { natural Serranía de los } \\
\text { Churumbelos Auka-Wasi }\end{array}$ & 2007 & Amazonía & $\begin{array}{l}\text { Cauca } \\
\text { Caquetá } \\
\text { Putumayo } \\
\text { Huila }\end{array}$ & \\
\hline $\begin{array}{l}\text { Parque nacional natural } \\
\text { Complejo Volcánico Doña } \\
\text { Juana-Cascabel }\end{array}$ & 2007 & $\begin{array}{l}\text { Andes (Nudo de } \\
\text { los Pastos) }\end{array}$ & $\begin{array}{l}\text { Cauca } \\
\text { Nariño }\end{array}$ & \\
\hline $\begin{array}{l}\text { Parque nacional natural } \\
\text { Yaigojé Apaporis }\end{array}$ & 2009 & Amazonía & $\begin{array}{l}\text { Vaupés } \\
\text { Amazonas }\end{array}$ & \\
\hline $\begin{array}{l}\text { Parque nacional natural } \\
\text { Uramba Bahía Málaga }\end{array}$ & 2010 & Costa del Pacífico & Valle del Cauca & \\
\hline $\begin{array}{l}\text { Parque nacional natural } \\
\text { Corales de Profundidad }\end{array}$ & 2013 & Costa del Caribe & Bolívar & \\
\hline $\begin{array}{l}\text { Parque nacional natural } \\
\text { Bahía Portete-Kaurrele }\end{array}$ & 2014 & Costa del Caribe & La Guajira & \\
\hline $\begin{array}{l}\text { Área natural única Los } \\
\text { Estoraques }\end{array}$ & 1988 & & & \\
\hline
\end{tabular}

Fuente: Elaboración propia.

\section{Algunas estrategias informativas y educativas para la sostenibilidad de espacios protegidos.}

Luego de haber conceptualizado las dimensiones informacionales y educativas como ejes para el abordaje de la sostenibilidad de los espacios ambientales protegidos y de identificar las zonas reconocidas en Colombia bajo criterios que las ubica en esa condición, vinculada con procesos de investigación científica, articulados a su vez con actividades turísticas responsables, mediadas por procesos educativos sobre el territorio; se proponen un conjunto de estrategias que integran tanto lo informacional como lo educativo.

En este sentido, el eje informacional de la propuesta plantea la posibilidad de diseñar, organizar y estructurar procesos y productos basados en la preparación de datos y contenidos sobre tales espacios ambientales protegidos. Se propone, como algunas de las actividades en el marco de este eje el desarrollo de sistemas de información que permitan el registro, inventario, documentación y catalogación de especímenes, piezas y elementos que hacen parte del territorio medioambiental propio de los espacios protegidos, 
como punto de partida para su estudio, comprensión y valoración. Esta actividad supone la realización de sinergias con diversos tipos de instituciones: Sistema Nacional de Áreas Protegidas (SINAP), bibliotecas especializadas, centros de documentación, universidades que cuentan con trabajos de investigación, relacionados con tales espacios protegidos, organizaciones científicas y otras entidades y corporaciones autónomas regionales vinculadas con el desarrollo sostenible en Colombia.

Esta estrategia requerirá de la evaluación de los recursos disponibles en la web sobre este tema, así como también de la actualización de la información relacionada con los espacios ambientales protegidos, con objeto de garantizar con un sistema actualizado alimentado permanentemente para ofrecer datos y contenidos relevantes sobre tales espacios ambientales. Además de la estructuración de sistemas de información de apoyo a los procesos de investigación, estudio y conocimiento de los espacios ambientales protegidos, también se debe realizar un trabajo mediante la incorporación de aplicaciones tecnológicas para profundizar en el conocimiento de los elementos y especímenes de gran valor, los cuales hacen parte del espacio ambiental. En este sentido, se considera pertinente la creación de senderos de información, y conocimiento, con la ayuda de herramientas tecnológicas, mediante cuya interacción sea posible profundizar en datos y contenidos para ampliar la compresión de tales espacios. La idea, es asumir que los espacios ambientales protegidos son hipertextos con enlaces informativos susceptibles de ser explorados para agregar valor a los contenidos disponibles. El ejercicio que se propone plantea un desplazamiento no solo a través de la apreciación de los espacios ambientales, sino también un desplazamiento considerando la información disponible sobre cada elemento que constituye tales territorios.

En cuanto al eje educativo, las estrategias tienen que ver con la inclusión en los planes de formación y actualización docente la dimensión medioambiental como patrimonio natural y como un tema que debe cruzar cual vector los asuntos del desarrollo humano integral, lo cual a su vez implica que se asuma a partir de los principios, objetivos y metas del desarrollo sostenible. La incorporación de este aspecto, como parte integrante de la formación de los docentes convoca también el desarrollo de estrategias transversales que implican incluir didácticas que busquen la generación de diálogos reflexivos con el 
entorno, la apreciación de los espacios protegidos en sus variadas aproximaciones: científica, patrimonial, estética y social, reconociendo la espacialidad como variable fundamental para el desarrollo. Los espacios ambientales protegidos también deben estar presentes en didácticas sensibilizadoras como los debates, cine-foros, las lecturas comentadas para afianzar los procesos de formación integral. Por ello, se cree que estos temas no solo deben hacer parte de los currículos específicos en donde tienen un lugar especial, la biología, la ecología, el patrimonio natural como variable esencial, sino que son contenidos que deben permear las diferentes mallas curriculares de las carreras que forman profesionales universitarios en las distintas áreas y campos del conocimiento.

El tema de la sostenibilidad de los espacios ambientales protegidos debe abordarse siguiendo estas y otras estrategias informativas y educativas, en virtud de que las actitudes de valoración del medioambiente pasan primero por la activación de procesos de apropiación, estudio, investigación, las cuales apoyan el conocimiento y la reconstrucción permanente de saberes y prácticas que contribuyan a entender lo medioambiental además de su dimensión crucial para la sostenibilidad también como documento vivo del que se pueden también desplegar procesos de conocimiento y reconocimiento del territorio como variable fundamental del desarrollo.

\section{Aportes conclusivos.}

La información y la educación son dos de los procesos-estrategias-componentes transversales que deben estar presentes en las propuestas de uso sostenible y ético del medioambiante, entendido como territorio de interacciones complejas entre actores sociales y los elementos que lo configuran, en tanto que espacio con potencialidad para la ciencia, la recreación y el reconocimiento de lo local y lo identitario. En el documento se pretendió caracterizar las dimensiones informativas y educativas como dos ejes medulares para abordar los espacios ambientales protegidos de Colombia, con objeto de considerarlos también desde los ámbitos científicos y culturales.

El componente informativo plantea todo un eje, en función del cual será posible pensar en estrategias para el registro, documentación y preparación de contenidos sobre tales espacios, como punto de 
partida para su estudio, comprensión y difusión, procesos estos que constituyen la base para avanzar hacia el reconocimiento de estos espacios como fuentes de información valiosas. El componente educativo alude a la necesidad de introducir estos temas en las propuestas de didácticas de todos los niveles y modalidades de los sistemas educativos. La idea es informar y educar para maximizar el aprovechamiento y apropiación simbólica y responsable de la riqueza que poseen estos espacios ambientales protegidos de Colombia, como estrategia fundamental para avanzar hacia la sostenibilidad del medioambiente y con ello las instituciones educativas y las unidades, servicios, sistemas y redes de información, mediante una sinergia creativa, contribuirán de forma decidida al logro de los objetivos del desarrollo sostenible. Por supuesto, la puesta en práctica de acciones que integren la información con la educación implica considerar un conjunto de factores, variables e indicadores que van desde contar con el personal debidamente formado para hacer realidad estas dinámicas, hasta las infraestructuras de procesos, contenidos y recursos que harán posible la creación de los entornos habilitadores que garantizarán su concreción en la práctica.

\section{Bibliografía}

Foronda Robles, C. (Coord.). (2012). Capital social en espacios naturales protegidos de España. Concepción Foronda Robles (Coord.) España: Ulzama. Disponible en https://rua.ua.es/dspace/bitstream/10045/25487 /1/Capital_Social.pdf

González Gaudiano, E. (1997). Educación Ambiental. Historia y conceptos a veinte años de Tbilisi..México: Sistemas Técnicos de Edición, S.A. de C.V

Gutiérrez Pérez, J. y Pozo Llorente, M. (2005) "Stultifera Navis: institucional tensions, conceptual chaos, and professional uncertainty at the beginning of the Decade of Education for Sustainable Development" en Policy Future in Education núm. 3, vol. 3, pp. 296-306

Hernández Llosas, M. (2002). Patrimonio cultural y desarrollo sostenible en la Quebrada de Humahuaca: Potencial y perspectivas. Cuadernos de la Facultad de Humanidades y Ciencias Sociales - Universi- 
dad Nacional de Jujuy, núm. 18, diciembre. San Salvador de Jujuy, Argentina.

Leal F, W. (2009) La educación para la sostenibilidad: iniciativas internacionales. Revista de Educación. Número extraordinario 2009. p-p 263-277. Recuperado de: http://www.revistaeducacion.mec.es/ re2009/re2009_12.pdf

Organización de las Naciones Unidas para la Educación, la Ciencia y la Cultura. (2014). Patrimonio cultural inmaterial y desarrollo sostenible. Disponible en: https://ich.unesco.org/doc/src/34299-ES.pdf

Ministerio de agricultura, alimentación y medio ambiente. (2012). Libro verde de sostenibilidad urbana y local en la era de la información. Madrid: España. Disponible en: http://www.mapama.gob.es/es/ calidad-y-evaluacion-ambiental/temas/medio-ambiente-urbano/ libro_verde_final_15.01.2013_tcm7 -247905. pdf

Ministerio de Ambiente, Vivienda y Desarrollo Territorial. Decreto No. 2372 de 2010. (2010) Recuperado de: http://www.minambiente.gov. co/images/normativa/decretos/2010/dec_2372_2010.pdf

Muñoz, (2006). Turismo y sostenibilidad en espacios naturales protegidos: La carta europea del turismo sostenible en la zona volcánica de la Garrotxa y el plan de desarrollo sostenible en Cabo de Gata-Nijar. Tesis doctoral: Universidad de Girona. Disponible en: http:// www.tesisenred.net/handle/ 10803/7913

P. Feinsinger, C. Pozzi, C. Trucco, R.L. Cuellar, A. Laina, M. Cañizares y A. Noss. (2010). Investigación, conservación y los espacios protegidos de América latina: una historia incompleta. Ecosistemas 19 (2): 97-111. Mayo 2010. España: Asociación Española de ecología terrestre. Disponible en: file:///C:/Users/yalmarza/Downloads/4994-1-SM.pdf

Ruiz, I., Barraza, y Paz Ceja, A. (2009). La educación para la sustentabilidad: análisis y perspectiva a partir de la experiencia de dos sistemas de bachillerato en comunidades rurales mexicanas. Recuperado de: file:///C:/Users/Invitado\%20SisInfo/Downloads/Dialnet-LaEducacionParaLaSustentabilidad-5026270\%20(1).pdf 
Sistema Nacional de áreas protegidas-SINAP. (S/F). Parques Nacionales naturales de Colombia. Disponible en: http://www.parquesnacionales.gov.co/ portal/es/sistema-nacional-de-areas-protegidas-sinap/

UNESCO (2006). "Education for Sustainable Development", Recuperado de: http://portal.unesco.org/education/

UNESCO (2014). El desarrollo sostenible comienza por la educación. Cómo puede contribuir la educación a los objetivos propuestos para después de 2015. Recuperado de: http://unesdoc.unesco. org/images/0023/002305/230508s.pdf

Unión interparlamentaria. (1989). Declaración de La Haya sobre el turismo. Disponible en: http://www.e-unwto.org/doi/pdf/10.18111/ unwtodeclarations. 1989.15.6.1

Vargas Larrubia, Remedios; Navarro Rodríguez, Susana; Luque Gil, Ana Ma. (2001). Desarrollo sostenible en espacios protegidos. La Comarca Malagueña de la Sierra de las Nieves. Cuadernos Geográficos, núm. 31, 2001 Universidad de Granada Granada, España, Disponible en: http://www.redalyc.org/articulo.oa?id=17103109 
